\title{
Quantifying Support
}

\author{
JOHN BLACK Malaspina College
}

\section{A. Introduction}

Teachers of informal logic and critical thinking have come to be concerned in recent years about the inadequacy of standard methods for assessing argument strength. The traditional notions of acceptability, relevance and sufficiency, while conceptually adequate, do not yield very fine-grained distinctions between arguments of varying strength. A number of philosophers have therefore initiated the search for a better system.

This theme was pursued at the Second Conference of the International Society for the Study of Argumentation, held in June 1990 at the University of Amsterdam. Stephen Thomas gave a presentation describing the system of adjectival gradations (strong, moderate, weak etc.) he uses in teaching the evaluation of arguments. The system is an elaboration on the one he has published in Practical Reasoning in Natural Language.' Mark Battersby went further, at least in intent: he gave a presentation calling for the investigation of quantitative methods for assessing degree of support, and hence the strength of arguments. He did not, however, provide details of a possible scheme for doing this, since his preliminary inquiries into its feasibility unearthed a number of difficulties.

In this paper I shall argue that, to the extent that it is plausible, Battersby's programme can be accomplished using a version of the well-known probability calculus. I shall provide an explanation of some of the complexities involved in applying the calculus to this programme, and a solution to some of the difficulties discovered by
Battersby. However, I shall also record my reservations about the generality of the application of the calculus to the assessment of argument strength.

\section{B. The Overall Strategy}

In applying the probability calculus to argument assessment, I shall make the assumption that what is required to judge argument strength is an assessment of the degree to which the initial premisses render the final conclusion acceptable. Determining this numerically will involve propagating acceptability through the support structure of the argument, beginning with the initial premisses, calculating their contribution of acceptability to the subconclusions, where these exist, and ultimately to the final conclusion.

Thus I shall propose a method in which the assigned acceptability-values of the initial premisses are used to calculate the values of what I shall call "received acceptability" for the remaining statements in the argument. The received acceptability of a conclusion may or may not be equal to its real acceptability: received acceptability is an assessment of degree of support, not of real acceptability. It sets the lower limit of acceptability for a conclusion, but no more.

I shall use the words "premiss" and "conclusion" to refer generally to the statements occurring in any inference-step in an argument, irrespective of whether they are initial premisses, final conclusions or subconclusions. For each inference-step, represented in the commonly-used tree diagram of the argument's structure by an 
arrow, the support given by the premiss(es) to the conclusion is a function of the acceptability of the premiss(es) and the sufficiency of the premiss(es) for the conclusion. In keeping with standard practice in informal logic, I shall use "acceptability" and "sufficiency" in such a way that the sufficiency of a premiss for a conclusion is independent of the acceptability of the premiss, and that of the conclusion.

\section{Probability and Acceptability}

For the sake of this paper, I shall more or less identify these notions. ${ }^{2}$ Both, of course, are open to a variety of interpretations and there are interesting parallels between disputes about each. Each notion can be treated as essentially objective, or as essentially subjective. These philosophical differences, however, do not impinge upon the applicability of the calculus in either case. I shall suppose that the calculus is equally applicable to acceptability and to probability, with one exception concerning conditional probability (see Section D).

Acceptability can be either assigned or received. The former is an absolute value more or less equivalent to the probability of the relevant statement's being true. The latter is a value relative to a particular argument, and to a stage in such an argument. It is a measure of the accumulated support for a statement, the degree to which those statements which precede the statement in the support-structure of the argument render the statement acceptable. Both types of acceptability are equivalent in the way they can be used to calculate the received acceptability of statements which follow them in the argument-structure.

The value of the acceptability of $p$ is written $A(p)$, in the case of assigned acceptability, and $A(p / \ldots)$ in the case of received acceptability, the lacuna being filled with a shorthand description of that from which $p$ is inferred. This value ranges between 0 and 1. Like probability, assigned accep- tability can be determined for truthfunctions. Thus,

(1)

(2) $A(p . q)=A(p) \times A(q)$

(3) $A(p \vee q)=A(p)+A(q)-A(p \cdot q)$

$=A(p)+A(q)-$ $(\mathrm{A}(\mathrm{p}) \times \mathrm{A}(\mathrm{q})) \quad$ by

(4) $A(p \rightarrow q)=A(\neg(p \cdot\urcorner q))$

$=1-A(p \cdot 7 q)$

$=1-(\mathrm{A}(\mathrm{p}) \mathrm{x}$ $A(-q))$

$=1-(\mathrm{A}(\mathrm{p}) \mathrm{x}$

$(1-A(q)))$

$=1-A(p)+(A(p) \times A(q))$

Some qualifications need to be noted. First, (2) is true only when $p$ and $q$ are independent of each other, and when they are not mutually exclusive, (If they are mutually exclusive, $A(p . q)=0$.) (2) is applicable, however, to all but the most contrived of examples, since the necessary conditions mentioned here are almost always fulfilled in any determination of the acceptability of a conjunction required by the present programme. The latter determination is restricted to the cases of linked premisses and, in one way of understanding the transmission of acceptability, of the conjunction of a premiss with the statement that the premiss entails a particular conclusion.

The second qualification concerns (3). The subtraction of the product of the two acceptabilities from their sum is justified in the following way. In determining the probability to be assigned to a disjunction, summing the probabilities of the two disjuncts leads to counting twice those circumstances in which both disjuncts are true. They are counted once in the truth-conditions for each disjunct. The result is a value for the probability of the disjunction which is inflated by an amount equal to the probability of both disjuncts being true. This amount must then be subtracted from the sum of the probabilities of the disjuncts. Since I am here identifying assigned acceptability and 
probability, the same result follows for the former notion.

Another way to look at this is to say that the assigned acceptability of the disjunction not being true is equal to that of both disjuncts being false. In other words,

$$
\begin{aligned}
& A(\neg(p \vee q))=A(\urcorner p \cdot \neg q) \\
& 1-A(p \vee q)=A(\neg p) \times A(\neg q)
\end{aligned}
$$

by (1) and (2)

$$
\begin{aligned}
A(p \vee q)= & 1-(A(\neg p) \times A(\neg q)) \\
= & 1-((1-A(p)) \times \\
& (1-A(q))) \quad b y(1) \\
= & 1-(1-A(p)- \\
& A(q)+(A(p) \times A(q))) \\
= & A(p)+A(q)-(A(p) \times \\
& A(q))
\end{aligned}
$$

It will be seen later (in Section E3) that this feature solves a problem identified by Battersby in his original presentation in Amsterdam.

As with (2), (3) applies only when $p$ and $q$ are independent of each other and not mutually exclusive; if they are mutually exclusive, there is no need to perform the final subtraction, since then $A(p . q)=0$. Like (2), (3) is applicable to all serious real-life arguments.

\section{Acceptability and Sufficiency}

To calculate the acceptability transmitted by a premiss to a conclusion (or, in other words, the received acceptability of the conclusion, relative to that premiss), the acceptability of the premiss is multiplied by its sufficiency for the conclusion. The latter is a value, also ranging between 0 and 1 , written as $S(p$ for $q)$, for premiss $p$ and conclusion $q$. The proposed way to handle the initial assignment of sufficiencies is to identify them with acceptabilities. There are three candidates for a way in which this might be done, but only one is appropriate.

The first is to use an analogue of conditional probability:

$$
\text { (6) } \mathrm{S}(\mathrm{p} \text { for } \mathrm{q})=\mathrm{A}(\mathrm{q}, \mathrm{p})=\frac{\mathrm{A}(\mathrm{q} \cdot \mathrm{p})}{\mathrm{A}(\mathrm{p})} \quad ? ? \text { ? }
$$

This route is not plausible in the present case. It is not at all clear how one could calculate a value for $\mathrm{A}(q . p)$, since $p$ and $q$ are not likely to be independent. If one were allowed to employ (2), this would make $\mathrm{A}(q, p)$ equal to $\mathrm{A}(q)$, creating two major problems. First, $\mathrm{A}(q)$ is unknown-it is what is to be determined through the entire quantification process. Second, if per impossibile it were known, the sufficiency of an inference would be identical to the acceptability of its conclusion, and this contradicts the required independence of the sufficiency of an inference-step and the acceptability of its premiss and conclusion. In so far as it applies to the quantitative assessment of support, the calculus has no need of conditional probability, and I shall assume that there is no defined analogue in the present application. ${ }^{3}$

Instead, I shall identify the sufficiency of an inference-step with the assigned acceptability of the corresponding conditional. The second candidate method to be rejected involves treating this conditional as a material conditional:
(7) $S(p$ for
$q)=A(p \rightarrow q)$
q) ???

This proposal also violates the required independence of sufficiency from the acceptability of premiss and conclusion, on account of the truth of (4). This leaves us with the third way, involving a stronger form of conditional, symbolized as:

(8) $\mathrm{S}(\mathrm{p}$ for $\mathrm{q})=\mathrm{A}(\mathrm{p} \longrightarrow \mathrm{p})$

When I talk of the conditional involved in the assessment of sufficiency, then, I mean it to be construed in terms of what I shall call "pragmatic implication." I must leave this notion intuitive for the moment. I do not want a form of conditional that is restricted to deductively valid inferences; I do want an analogue of deductive entailment which preserves one of its features, but which can be applied to inferences of all kinds. ${ }^{4}$ Just as whether or not $p$ entails $q$, for any $q$, is independent of the truth of $p$, the sufficiency of $p$ for any $q$ is to be 
independent of the acceptability of $p$. I shall refer to such conditionals in this context as "connecting conditionals."

The substitution of the acceptability of a certain conditional for the sufficiency of an inference-step can be seen as equivalent to recasting the argument in deductively valid form. Corresponding to each inference-step is a conditional, which together with the premiss entails the conclusion. The acceptability transmitted to the conclusion is the acceptability of the conjunction of the premiss and the conditional. Since premiss and conditional are mutually independent, this is equal to the product of their acceptabilities.

\section{E. How to Make the Numbers Work}

As I said above, the acceptability received by $q$ from the single premiss $p$ is the product of the acceptability of $p$ and the sufficiency of $p$ for $q$. Hence,

$$
\begin{aligned}
A(q / p) & =A(p) \times S(p \text { for } q) \\
& =A(p) \times A(p-\longrightarrow q), \text { by }(8)
\end{aligned}
$$

or, more generally,

$$
\text { (10) } \begin{aligned}
\mathrm{A}(\mathrm{q} / \mathrm{p} / \ldots) & =\mathrm{A}(\mathrm{p} / \ldots) \times \mathrm{S}(\mathrm{p} \text { for } \mathrm{q}) \\
& =\mathrm{A}(\mathrm{p} / \ldots) \times \mathrm{A}(\mathrm{p}-\longrightarrow \mathrm{q})
\end{aligned}
$$

What this means is that the acceptability received by $q$ from $p$ is equal to the acceptability of the conjunction of $p$ and $(p-D>q)$.

Apart from simple support, the calculus has to be able to handle serial, linked and convergent support. (In this context, divergent support is a trivial embellishment of simple support.)

\section{E1. Serial Support}

For premiss $p$, subconclusion $q$ and conclusion $r$,

$$
\text { (11) } \begin{aligned}
\mathrm{A}(\mathrm{r} / \mathrm{q} / \mathrm{p})= & \mathrm{A}(\mathrm{q} / \mathrm{p}) \mathrm{x} \\
& \mathrm{S}(\mathrm{q} \text { for } \mathrm{r}) \\
= & \mathrm{A}(\mathrm{p}) \times \mathrm{S}(\mathrm{p} \text { for } \mathrm{q}) \\
& \times \mathrm{S}(\mathrm{q} \text { for } \mathrm{r})
\end{aligned}
$$

$$
\begin{aligned}
= & A(p) \times A(p-D\rangle q) \\
& \times A(q-D r)
\end{aligned}
$$

or, generally,

$$
\text { (12) } \begin{aligned}
\mathrm{A}(\mathrm{r} / \mathrm{q} / \mathrm{p} / \ldots)= & \mathrm{A}(\mathrm{q} / \mathrm{p} / \ldots) \times \mathrm{S}(\mathrm{q} \text { for } \mathrm{r}) \\
= & \mathrm{A}(\mathrm{p} / \ldots) \times \mathrm{S}(\mathrm{p} \text { for } \mathrm{q}) \\
& \times \mathrm{S}(\mathrm{q} \text { for } \mathrm{r}) \\
= & \mathrm{A}(\mathrm{p} / \ldots) \times \mathrm{A}(\mathrm{p}-\longrightarrow \gg \mathrm{q}) \\
& \times \mathrm{A}(\mathrm{q}-\gg \mathrm{r})
\end{aligned}
$$

\section{E2. Linked Support}

Linked support is handled by conjoining the premisses and calculating the product of their acceptabilities and that of the connecting conditional whose antecedent is their conjunction.

$$
\begin{aligned}
S(p+q \text { for } r)= & A((p . q)-\gg r) \\
A(r / p+q)= & A(p) \times A(q) \times \\
& A((p . q)-\gg r)
\end{aligned}
$$

or, generally,

$$
\begin{gathered}
\text { (15) } \mathrm{A}(\mathrm{r} /(\mathrm{p} / \ldots)+(\mathrm{q} / \ldots))=\mathrm{A}(\mathrm{p} / \ldots) \times \\
\mathrm{A}(\mathrm{q} / \ldots) \times \mathrm{A}((\mathrm{p} . \mathrm{q})-\longrightarrow \mathrm{r})
\end{gathered}
$$

\section{E3. Convergent Support}

The convergent case is slightly more complicated, and almost led Battersby to despair of his programme during the Amsterdam presentation. If a conclusion is supported by two independent premisses, we want to sum the contributions of each, and yet this can lead to acceptabilities greater than 1, which contradicts our rules for assigning acceptabilities. The answer to the conundrum is provided, however, by the manner in which the probability calculus handles disjunction. In the simple case, the received acceptability of a conclusion $r$ supported by two convergent premisses $p$ and $\mathrm{q}$ is given by:

$$
\text { (16) } \begin{aligned}
\mathrm{A}(\mathrm{r} / \mathrm{p} ; \mathrm{q})= & \mathrm{A}(\mathrm{r} / \mathrm{p})+\mathrm{A}(\mathrm{r} / \mathrm{q})- \\
= & (\mathrm{A}(\mathrm{r} / \mathrm{p}) \times \mathrm{A}(\mathrm{r} / \mathrm{q})) \\
& (\mathrm{A}(\mathrm{q}) \times \mathrm{A}(\mathrm{p}->\mathrm{q}))+ \\
& -(\mathrm{A}(\mathrm{p}) \times \mathrm{A}(\mathrm{p})) \\
& \times(\mathrm{A}(\mathrm{q}) \times \mathrm{A}(\mathrm{q}--\gg \mathrm{r})))
\end{aligned}
$$

or, in the general case, 


$$
\text { (17) } \begin{aligned}
& A(r /(p / \ldots) ;(q / \ldots)) \\
&= A(r / p / \ldots)+A(r / q / \ldots)-(A(r / p / \ldots) \\
&x A(r / q / \ldots)) \\
&=(A(p / \ldots) \times A(p-\gg r))+ \\
&(A(q / \ldots) \times A(q-\gg r)) \\
&-((A(p / \ldots) \times A(p-\gg))) \times \\
&(A(q / \ldots) \times A(q-\gg r)))
\end{aligned}
$$

This equation indicates that the acceptability received by $r$ from the two independent premisses $p$ and $q$ is equivalent to the acceptability of the disjunction of the conjunctions p. $(\mathrm{p} \longrightarrow \mathrm{r})$ and $\mathrm{q} \cdot(\mathrm{q}-\longrightarrow \mathrm{r})$. The subtraction of the product of the two contributions ensures that the acceptability received by the conclusion never rises above 1 .

The same principle governs the formula for cases involving more than two convergent premisses. In each case we calculate the acceptability of the disjunction of the conjunctions of each premiss with its connecting conditional. The precise mathematics are too detailed to be repeated here, but it can be seen that consideration of the process used to generate (3) from (5) yields:

$$
\text { (18) } \begin{aligned}
\mathrm{A}(\mathrm{r} /(\mathrm{p} / \ldots) ; \ldots ;(\mathrm{z} / \ldots))=1- \\
((1-\mathrm{A}(\mathrm{r} / \mathrm{p})) \times \ldots \ldots \times(1-\mathrm{A}(\mathrm{r} / \mathrm{z})))
\end{aligned}
$$

Before I move on, I wish to make a brief note about degrees of significance in relation to the calculation of received acceptability. The value calculated for the received acceptability of a conclusion should be stated to a number of significant figures no greater than the smallest number of significant figures occurring in the assignment of acceptabilities to premisses and connecting conditionals. This constraint is derived from some general considerations in error theory which it would be tedious to elaborate here. It is a moot point, for me, whether the rounding should take place only at the end of the propagation or at each stage in the argument-structure. I should recommend the first, but am unsure of the principles which might justify this recommendation.

\section{F. Counter-considerations}

At Amsterdam, Battersby expressed the hope that any quantitative sy tem developed for argument evaluation would be able to handle counter-considerations. The present one has that ability. To demonstrate this, I shall examine counter-considerations of two kinds: (1) those directed at a statement in the argument, and (2) those directed at an inference-step.

\section{F1. Statement-counters}

Consider the simple case of a counterconsideration $q$ directed at a conclusion $r$. All that needs to be done in this case is to reduce the acceptability received by $r$ from the rest of the argument up to that stage by an amount proportional to the acceptability given by $q$ to $7 r$. If $A(r / \ldots)$ is the initial value calculated for the acceptability of $r$ from the positive support for $r$, then the adjusted value is given by:

$$
\text { (19) } \begin{aligned}
\mathrm{A}(\mathrm{r} / \ldots \backslash \mathrm{q})= & \mathrm{A}(\mathrm{r} / \ldots)-(\mathrm{A}(\mathrm{r} / \ldots) \times \\
& \mathrm{A}(\neg \mathrm{r} / \mathrm{q})) \\
= & \mathrm{A}(\mathrm{r} / \ldots) \times(1-\mathrm{A}(\neg \mathrm{r} / \mathrm{q}))
\end{aligned}
$$

$A(\neg r / q)$ is of course given by:

(20) $A(\neg r / q)=A(q) \times A(q-\longrightarrow r)$

Another way to understand (19) is to reflect that if the counter-consideration $q$ were true, and if the conditional $q-\gg r$ were also true, then $r$ could not be true. The support for $r$ is thus contingent upon the falsity of the conjunction of the counter and its connecting conditional. To assess the value of that support, we need to consider the acceptability of the conjunction of the assertion of the conjunction of the positive premiss and its connecting conditional together with the negation of the conjunction of the counter and its connecting conditional. Take the simple case where the positive support for $r$ comes from the single premiss $p$ :

$$
\text { (21) } \begin{aligned}
\mathrm{A}(\mathrm{r} / \mathrm{p} \backslash \mathrm{q})=\mathrm{A}((\mathrm{p} \cdot(\mathrm{p}-\gg \mathrm{r})) \\
\neg(\mathrm{q} \cdot(\mathrm{q}-\gg \mathrm{r})))
\end{aligned}
$$




$$
\begin{aligned}
= & A(p \cdot(p--) r)) x \\
& A(\neg(q \cdot(q->))) \\
= & A(p \cdot(p-b r)) \times \\
& (1-A(q \cdot(q->r))) \\
= & A(r / p) \times(1-A(-r / q))
\end{aligned}
$$

The last line above can be generalized to yield (19).

In more complicated cases, the value of $A(7 \mathrm{q} / \mathrm{p})$ may have to be calculated in complicated ways, depending on whether there are many linked or convergent counterconsiderations, for example. The rule of thumb is to combine the counterconsiderations into one and then apply (19).

\section{F2. Inference-counters}

An argument may countenance a consideration which seems to undermine not one of its statements, but instead one of its inferences. In this case, we need to adjust the value of the sufficiency accorded to the relevant inference-step. In the case of a counter $q$ directed at the inference from $p$ to $r$,

(22) $S(p$ for $r \backslash q)=S(p$ for $r)-(S(p$ for $r)$ $x A(\neg(p-\gg r) / q))$

or, by $(8)$,

$$
\begin{aligned}
A(p-\gg r \backslash q)= & A(p-\gg r)- \\
& (A(p-\gg r) \times \\
& A(\neg(p-\gg r) / q)) \\
= & A(p-\gg r) \times(1- \\
& A(\neg(p-\gg r) / q))
\end{aligned}
$$

where,

$$
\begin{aligned}
& A(\neg(p-D r) / q)=A(q) x \\
& A(q-\gg(p-D r))
\end{aligned}
$$

Once again, (23) can be read as saying that the acceptability of the connecting conditional, in light of the counter-consideration, is equal to the acceptability of the conjunction of the connecting conditional with the negation of the conjunction of the counter and its connecting conditional,
Before we leave the topic of counterconsiderations, it is worthwhile to mention a general constraint on the assignment of values to the acceptability of counters. This is summarised, in the case of a counter $p$ to a conclusion $q$, by:

(25) $\mathrm{A}(\mathrm{q} / \ldots)+\mathrm{A}(7 \mathrm{q} / \mathrm{p}) \leq 1$

This is so because the received acceptabilities of each of $q$ and $q$ are the lower bounds of their real acceptabilities, and the latter sum to 1.

\section{G. Thomas's Adjectives}

As I mentioned earlier, Stephen Thomas also made a presentation in Amsterdam. He argued that his system of assessing argument strength had proved to be successful in classroom trials, and had enabled his students to evaluate many of Hume's arguments, for example, without prior exposure. Thomas's method applies, so far, only to single inferences, whether simple or linked. Like the method proposed here, it also involves combining an assessment of acceptability with one of sufficiency to reach one of argument "soundness and reliability." Unlike those employed in the present quantitative method, these assessments are not expressed in numerical terms.

According to material distributed by Thomas at Amsterdam, what 1 call acceptability is evaluated using the terms "definitely true," "probably true,", "uncertain/don't know," "probably false", and "definitely false." What I call sufficiency (Thomas prefers "validity") is expressed using the terms "deductively valid," "strong," "moderate," "can't tell," "weak" "and "nil." The strength of the argument, or reasoning, is expressed using the terms " $\mathrm{S}$ : sound and reliable," "PS: probably sound and reliable," "M: marginal" ( = close to sound and reliable, but not quite certain or totally reliable beyond a reasonable doubt), "CS: cannot say," "PU: probably unsound and 
unreliable" and " $U$ : unsound and unreliable." For brevity, I shall use "sound" instead of "sound and reliable," and so forth.

I propose to test Thomas's intuitions about degrees of argument strength by using part of my method, the part which involves multiplying acceptability-values by sufficiency-values to reach a numerical assessment of argument strength. (These intuitions, of course, may or may not accord with my own or with the reader's.) To do this I'll use a "spectrum of validity" diagram provided by Thomas at Amsterdam (a similar diagram appears on p.135 of his textbook mentioned above) to assign values of sufficiency (or validity, in his terminology), as follows:

$\begin{array}{lll}\text { Deductively valid } & =1 \\ \text { Strong } & =0.95-0.99 \\ \text { Can't tell (rare) } & =0.5 \\ \text { Moderate } & =0.53-0.94 \\ \text { Weak } & =0.01-0.52 \\ \text { Nil } & =0\end{array}$

These values, aside from the first, third and last, are based on a linear measure of Thomas's diagram. The diagram is rough: it is possible that Thomas intended the border between "moderate" and "weak" to fall at 0.5 , for example, but otherwise the level of accuracy is sufficient for my purposes. The first and last values are obvious; the third just gives the best estimate in a case where nothing inclines one either way on the judgment of sufficiency.

Assigning acceptability values is a bit more complex, but here goes:

$\begin{array}{lll}\text { Definitely true } & =1 \\ \text { Probably true } & =0.75-0.99 \\ \text { Don't know } & =0.26-0.74 \\ \text { Probably false } & =0.01-0.25 \\ \text { Definitely false } & =0\end{array}$

The problems here are the "probably" statements. Thomas defines "probably true/false" as "it is highly likely that this statement is true/false, but I do not know this with complete certainty." I was tempted to assign ranges identical to that for "strong," by analogy with his spectrum for validity, where, for example, he defines "strong" as:

If the reasons were true, they would make the truth of the conclusion extremely likely, certain beyond any reasonable doubt, "virtually a sure thing," but not totally guaranteed. Likely enough to make it reasonable to stake something of great value on the truth of the conclusion if the reasons are true, and likely enough to serve as a definitely reliable basis for actions. ${ }^{5}$

It's arguable, though, that this second definition is more restrictive than the firstthough not entirely so. I have thus extend. ed the ranges. I also wished to ensure that there are no gaps in the scheme for acceptability. Hence the broad range for "don't know."

For the sake of argument, I shall take values in the middle of each range as representative of the range. Thomas provides the following matrix for judgments of argument strength. I have amended it by writing in the values for premissacceptability, inference-sufficiency and argument strength, as determined by their product, the received acceptability of the conclusion:

TABLE OF SOUNDNESS AND RELIABILITY VERDICTS ${ }^{5}$

\section{DEGREE OF VALIDITY \\ (DEGREE OF CONFIRMATION OF THE INFERENCE)}

\begin{tabular}{|c|c|c|c|c|c|c|}
\hline $\begin{array}{l}\text { STATUS } \\
\text { OF THE } \\
\text { REASONS }\end{array}$ & $\begin{array}{c}\text { Ded } \\
\text { Valid } \\
1\end{array}$ & \begin{tabular}{|c|} 
Strong \\
0.97
\end{tabular} & \begin{tabular}{|l} 
Can't \\
Tell \\
0.5 \\
\end{tabular} & $\begin{array}{l}\text { Mod- } \\
\text { erate } \\
0.74 \\
\end{array}$ & $\begin{array}{l}\text { Weak } \\
0.26\end{array}$ & $\begin{array}{c}\text { Nil } \\
0 \\
\end{array}$ \\
\hline $\begin{array}{l}\text { Def } \\
\text { true }\end{array}$ & $\begin{array}{l}S \\
1\end{array}$ & $\begin{array}{c}S \\
0.97\end{array}$ & $\begin{array}{l}\mathrm{CS} \\
0.5\end{array}$ & $\begin{array}{c}\mathrm{U} \\
0.74 \\
\end{array}$ & $\begin{array}{c}\mathrm{U} \\
0.26 \\
\end{array}$ & $\begin{array}{l}u \\
0\end{array}$ \\
\hline $\begin{array}{l}\text { Prob } \\
\text { true } 0.87\end{array}$ & \begin{tabular}{|c|} 
PS \\
0.87
\end{tabular} & $\begin{array}{c}M \\
0.84\end{array}$ & $\begin{array}{c}\mathrm{CS} \\
0.44\end{array}$ & $\begin{array}{c}\mathrm{U} \\
0.64\end{array}$ & $\begin{array}{c}\mathrm{U} \\
0.23\end{array}$ & $\begin{array}{l}U \\
0\end{array}$ \\
\hline $\begin{array}{l}\text { Don't } \\
\text { know } 0.5\end{array}$ & $\begin{array}{l}\text { CS } \\
0.5\end{array}$ & $\begin{array}{c}\text { CS } \\
0.49\end{array}$ & $\begin{array}{c}\mathrm{CS} \\
0.25\end{array}$ & $\begin{array}{c}\mathrm{U} \\
0.37\end{array}$ & $\begin{array}{c}U \\
0.13\end{array}$ & $\begin{array}{l}U \\
0\end{array}$ \\
\hline $\begin{array}{l}\text { Prob } \\
\text { false } 0.13\end{array}$ & $\begin{array}{l}\mathrm{PU} \\
0.13\end{array}$ & $\begin{array}{l}\text { PU } \\
0.13\end{array}$ & $\begin{array}{c}\text { PU } \\
0.07\end{array}$ & $\begin{array}{l}\mathrm{U} \\
0.1\end{array}$ & $\begin{array}{c}\mathrm{U} \\
0.03\end{array}$ & $\begin{array}{l}\mathrm{U} \\
0\end{array}$ \\
\hline $\begin{array}{l}\text { Def } \\
\text { false }\end{array}$ & $\begin{array}{l}U \\
0\end{array}$ & $\begin{array}{l}\mathrm{U} \\
0\end{array}$ & $\begin{array}{l}U \\
0\end{array}$ & $\begin{array}{l}\mathrm{U} \\
0\end{array}$ & $\begin{array}{l}\mathrm{U} \\
0\end{array}$ & $\begin{array}{l}\mathrm{U} \\
0\end{array}$ \\
\hline
\end{tabular}


Examination of the numerical matrix reveals that in several areas there is a clash of intuitions. Of course this may be due to the artificiality of my assignments of values to Thomas's adjectives, especially in the area of acceptability, but it may also be a result of genuine differences in subjective estimates of argument strength.

The first area of concern is the estimate of argument strength when the premisses are either definitely true or probably true and the reasoning is moderately valid. My method (in company with my intuitions) indicates that Thomas's assessment of "unsound" is rather harsh, since the corresponding values of received acceptability for the conclusion are 0.74 and 0.64 . Whatever value we take within the range assigned to a moderate degree of validity, the argument strength would be greater than 0.5 in all cases when the premisses are definitely true, and in many when they are merely probably true. In many cases the estimate of argument strength in these categories will be greater than 0.9 , given that moderate validity extends up to sufficiency-values of 0.94 . I think Thomas is a more conservative gambler than I!

I should, however, stand behind my intuitions in these cases. Thomas's verbal description of moderate validity is:

\footnotetext{
Less than "strong" but more than "weak." If the reasons were true, they would not establish the truth of the conclusion as "a sure thing," but they would at least make it a "good bet." However, more would be required to establish the conclusion beyond any reasonable doubt. ${ }^{5}$
}

Thomas's table of soundness verdicts seems a little at odds with this description of the acceptability of the conclusion in the case when the premisses are true, and in fact this description seems more in accord with the values assigned by my method.

The second area of concern is the one in which the premisses are probably false, though the reasoning is deductively valid, strong or unknown. In these cases, Thomas's judgments of "probably unsound" seem rather lenient when compared with the numerical values generated by my method. This impression is magnified when a comparison is made with the first area of concern. I suggest that Thomas's intuitions are influenced to an inappropriate degree by the validity-factor and insufficiently by the premiss-factor. Could they be the intuitions of one who, like me, has devoted many years to trying to get students to acknowledge the importance of the concepts of inference-strength (validity, sufficiency) to the evaluation of arguments? My method, based heavily on acceptability, kowtows to the undergraduate fixation with issues of truth and falsity, but as a result produces what I think is a more balanced table of soundness verdicts.

The final area of concern is the verdict when the premisses are probably true ("highly likely") and the reasoning is strong. Thomas's soundness verdict of "marginal" seems a little harsh when one considers that the only form of validity which is stronger than "strong" is deductive validity, and "probably true" includes all strong likelihoods except absolute certainty. The value assigned by my method reflects my intuition that the reasoning is at least "probably sound."

Thus I should be prepared to defend my method against the apparent deviations from Thomas's intuitions which it necessitates. I should remind the reader once again, however, that the initial assignment of values upon which the consideration of Thomas's table is based is provisional to a great extent. I am sure that refinements could be suggested. Nevertheless, I think that the approach I have taken is vindicated by a comparison with Thomas's parallel but non-quantitative approach. The comparison suggests to me that the precision of the numerical assessment of argument strength provides a certain level of objectivity. Inherent in the relative imprecision of the adjectival approach is a temptation to subjectivity, for which my method can be seen as an antidote. 


\section{H. Reservations}

Having spent some time developing and motivating the above method for the quantitative assessment of argument strength, I now wish to record my strong reservations about its general applicability. The problem lies, I think, not in the calculation of the way in which acceptability can be propagated through an argument's structure, but in the initial assignment of acceptabilityvalues both to premisses and to connecting conditionals.

Certain assignments are noncontroversial and these, I suppose, guarantee the method some application, even if a limited one. Included in this set of assignments will be cases of known truth and falsity (values of 1 or 0 ), though in these cases the use of the calculus method will likely be both trivial and burdensome. Also included will be the application to probability-statements, where fractional values gain a foothold. Arguments all of whose premisses and connecting conditionals fall into these two classes of statement will then be non-controversially evaluable using the quantitative method.

I am not confident, however, that it will be applicable in any other cases. The difficulty of assigning "accurate" values to the acceptability of statements in general is not an epistemic difficulty, but a conceptual one. What does it mean to say that the acceptability-value of "Brian Mulroney's days in office are numbered" is 0.93 ? Not a lot, it seems to me. At most, the numerical value could be treated as an estimate of the subjective degree of confidence which the argument-evaluator holds in the truth of the statement. Here it is hard to see that it makes sense to specify the value beyond the first significant figure-our intuitions are just not that fine-grained, which is why Thomas's method is far more intuitively appealing.

Still, it might be thought, even this limited approach might be useful in testing and correcting our unquantified intuitions about argument strength. I have used the method to this end in examining Thomas's table of soundness verdicts. Employed in this way, the quantitative method seems to be a useful tool in the workshop of argument evaluation. Its use, however, is largely negative: I think it would seldom, if ever, be plausible to prefer the quantitative result to one's own thought-out intuitions.

In any real case of conflict between intuitive and quantitative assessments of argument strength, I would be inclined to let my intuitions about argument strength overrule the result dictated by the quantitative method. Rather than admit that my intuitions were wrong, I would go back and alter the initial assignments of acceptability to the premisses or connecting conditionals so that the result comes out more in line with my intuitions. This is not stubbornness-the point is that I am likely to be more certain in my estimate of argument strength than I am in my assignment of initial acceptabilities. (My attitude here is parallel to the one I should evince if a moral theory which I thought I held dictated an intuitively unpalatable verdict on a specific case of moral action.)

My conclusion, then, is a modest one: to the limited extent that a quantitative method for assessing argument strength is viable, it should take the form described herein. Having spent some effort in developing the method, I should be delighted if someone could suggest a class of applications for which it is really useful, but that task I must leave to minds of a bent more formal than my own.

\section{Notes}

1 Stephen Thomas, Practical Reasoning in Natural Language, Englewood Cliffs, Prentice-Hall, 1986.
2 In his book Plausible Reasoning, Amsterdam. Van Gorcum, 1976, Nicholas Rescher investigates in a quasi-quantitative way the 
notion of plausibility. Some passages appear to suggest, though this is not entirely clear, that he thinks the latter notion is to be preferred to that of probability in assessing the acceptability of a conclusion drawn from its premisses. Space does not allow for a full treatment of this interesting claim, but I shall offer one comment here. It is a feature of Rescher's account of plausibility that a conclusion (of a deductive argument) is no more and no less plausible than its least plausible premiss; this distinguishes plausibility from probability. It has the consequence, however, that a conclusion gains no increase in plausibility when a premiss other than the least plausible of the premisses is discovered to be more plausible than originally thought. In my view, this feature prevents conclusionplausibility from being considered an adequate assessment of argument strength, as premissplausibility from being an adequate assessment of premiss-acceptability. For if Rescher's account were applicable to the case imagined here, an increase in premiss-acceptability would produce no increase in argument strength.
3 This may be unduly pessimistic. At the very least it might be possible to employ the notion of conditional acceptability, defined in terms of real acceptabilities, for purposes other than the definition of sufficiency. For an investigation of similar possibilities in relation to the special case of plausible reasoning in a deductive context, see George Polya, Mathematics and Plausible Reasoning, Princeton, Princeton University Press, 1954. Polya's notion of plausible reasoning differs from Rescher's (see previous note). Polya is concerned with the increase in the plausibility of a conjecture when one of its deductive consequences is found to be true.

4 For similar declarations about this important notion, see Jonathan Berg, "Interpreting Arguments, " Informal Logic IX. 1, 1987, and Trudy Govier, A Practical Study of Argument, Belmont, Wadsworth, 1985 , p. 60 ff.

5 These passages are all taken from material handed out by Thomas at his presentation in Amsterdam. Similar formulations occur in his textbook.

\section{Appendix: Explanation of Symbols}

\begin{tabular}{|c|c|}
\hline $\mathrm{p}$ & $=$ the negation of $p=$ not $-p$ \\
\hline p.q & $=$ the conjunction of $p$ and $q$ \\
\hline & $=$ the disjunction of $p$ and $q=p$ or $c$ \\
\hline$q$ & $=$ if $\mathrm{p}$ then $\mathrm{q}$ (material conditional) \\
\hline$-\rightarrow q$ & $=$ if $p$ then $q$ (pragmatic implication) \\
\hline $\mathrm{A}(\mathrm{p})$ & $=$ the assigned acceptability of $p$ \\
\hline$A(p / q)$ & $\begin{aligned}= & \text { the received acceptability of } p \text {, } \\
& \text { relative to } q\end{aligned}$ \\
\hline$A(p / \ldots)$ & $\begin{array}{l}=\text { the received acceptability of } p \text {, } \\
\text { relative to whatever precedes } p \text { in } \\
\text { the argument-structure }\end{array}$ \\
\hline$A(p / q / r)$ & $\begin{array}{l}=\text { the received acceptability of } p \text {, } \\
\text { relative to } q \text {, which is preceded in } \\
\text { the argument-structure by } r\end{array}$ \\
\hline $19+$ & $\begin{aligned}= & \text { the received acceptability of } p \text {, } \\
& \text { relative to linked premisses } q \text { and } r\end{aligned}$ \\
\hline
\end{tabular}
$\mathrm{A}(\mathrm{p} / \mathrm{q} ; \mathrm{r}) \quad=$ the received acceptability of $\mathrm{p}$, relative to convergent premisses $q$ and $r$
$A(p \backslash q)=$ the assigned acceptability of $p$, despite counter-consideration $\mathrm{q}$
$A(p / q \backslash r)=$ the received acceptability of $p$, relative to $q$, despite counter- consideration $r$

$S(p$ for $q)=$ the sufficiency of $p$ for $q$

$S(p$ for $q \backslash r)=$ the sufficiency of $p$ for $q$, despite counter-consideration $\mathbf{r}$

JOHN A. BLACK

PHILOSOPHY DEPARTMENT

MALASPINA COLLEGE

900 FIFTH STREET

NANAIMO, B.C. V9R $5 S 5$ 